\title{
Renal Mucinous Tubular and Spindle Cell Carcinoma: A Report of Two Cases and Literature Review
}

\author{
Giulia de Assis Queiroz ${ }^{1}$, Rafael Shiguetaro Lemos Sudo' ${ }^{1}$, Thales Junqueira Oliveira ${ }^{1}$, William Nicoleti Turazza da Silva ${ }^{1}$, Bruno \\ de Carvalho Dornelas ${ }^{1,2 *}$
}

${ }^{1}$ Medical School, Federal University of Uberlandia, Minas Gerais, Brazil

2Pathology Department, Clinic's Hospital, Federal University of Uberlandia, Minas Gerais, Brazil

*Corresponding author: Bruno de Carvalho Dornelas, Pathology Department, Clinic's Hospital, Federal University of Uberlandia, Minas Gerais, Brazil; Tel: +553432182263, Fax: +553432258621; E-mail: bruno.dornelas@ufu.br

Received: July 17, 2019; Accepted: July 26, 2019; Published: August 11, 2019;

\begin{abstract}
Mucinous tubular and spindle cell carcinoma (MTSCC) is a rare type of epithelial renal tumor considered as a low-grade neoplasm by the World Health Organization. Therefore, partial or radical nephrectomy may be curative whether in early stages. Less than a hundred of these tumors have been reported with gross and microscopic features, immunohistochemical profile and clinical findings. Here we report two cases of MTSCC with emphasis on the clinicopathological correlations according to postoperative findings. Both patients underwent a video-assisted resection of the kidney. No recurrence or metastasis was observed during the follow-up.
\end{abstract}

Keywords: Renal Mucinous Tubular and Spindle cell Carcinoma, Renal Cell Carcinoma, Pathology

\section{Introduction}

Mucinous tubular and spindle cell carcinoma (MTSCC) is a histological type of renal carcinoma endorsed as an entity in 2004 by the World Health Organization (WHO) [1]. It accounts for less than $1 \%$ of all kidney tumors and shows a higher prevalence in women. Until 2015, there were less than 100 cases reported in the literature [26]. MTSCC may be associated with monosomy 1, 4, 6, 8, 13 or 14 and trisomy 7, 11, 16 or 17. Microscopically, the neoplasm is characterized tubular growth pattern of uniform cuboidal cells with eosinophilic cytoplasm which transition to anastomosing spindle cells in a myxoid and bubbly with abundant extracellular mucin stroma [1,2,4-11]. MTSCC is a low malignant potential cancer that rarely relapses or metastasizes [5,12].

We present two cases of mucinous tubular and spindle cell carcinoma diagnosed and treated in a high complexity Brazilian hospital.

\section{Case Presentation}

\section{Case One}

A 61-year-old, previously healthy woman attended to the urology outpatient clinic due to an incidental image finding. An abdominal CT scan had shown a $2-\mathrm{cm}$ nodular mass in the upper pole of the left kidney. The physical examination was normal. Laboratory studies and renal function tests showed no changes. Video-assisted left radical nephrectomy and perihilar lymphadenectomy were performed. The gross examination revealed a well-circumscribed grayish-white tumor confined to the atrophic kidney (figure 1). No renal vein invasion was detected. Microscopic examination of the mass showed low nuclear grade cells with focally vacuolated eosinophilic cytoplasm and spindle cells organized in long tubular profiles. Some clusters of foamy macrophages were present and extracellular mucin was highlighted by Alcian blue stain (figure 2). No sarcomatoid features were seen. Immunohistochemistry reactions were performed and the cells stained for CK7 and alphamethylacyl-CoA racemase (AMACR) and showed negativity for vimentin and CD10 (table 1). In conclusion, the diagnosis of a mucinous tubular and spindle cell carcinoma was stated. No metastasis was present in the lymph node submitted for evaluation. The patient has been on follow-up for 2 years and had no complaints. Imaging exams have not detected abnormalities.

\section{Case Two}

A 71-year-old woman was admitted in the Emergency Room. She has had moderate left flank pain that radiated to the chest and left leg over the past 6 months. On a scale of 1 to 10 , the pain was 6 . She also complained about dysuria. No weight-loss had been noticed. She had a long-term smoking history and had received irregular treatment for systemic arterial hypertension. On physical examination, her temperature was normal and blood pressure was 140/90 mmHg. There was pain on deep left flank palpation. An abdominal ultrasound showed a $4.7-\mathrm{cm}$ nodular mass suspected of malignancy in the left kidney. A partial video-assisted nephrectomy was performed. On the specimen's gross examination, a pushing border nodular tumor was found. It measured up to $3.8 \mathrm{~cm}$ in diameter and was yellowish. No renal vein invasion was detected. Microscopic examination of the 
tumor revealed Fuhrman grade 1 nucleus low cuboidal and spindle cells arranged in tubular or cord-like growth pattern (figure 3). Stroma was myxoid and bubbly (figure 4). No sarcomatoid features were present. Immunohistochemistry reactions were performed and the cells stained for CK7 and AMACR and vimentin (table 1). The

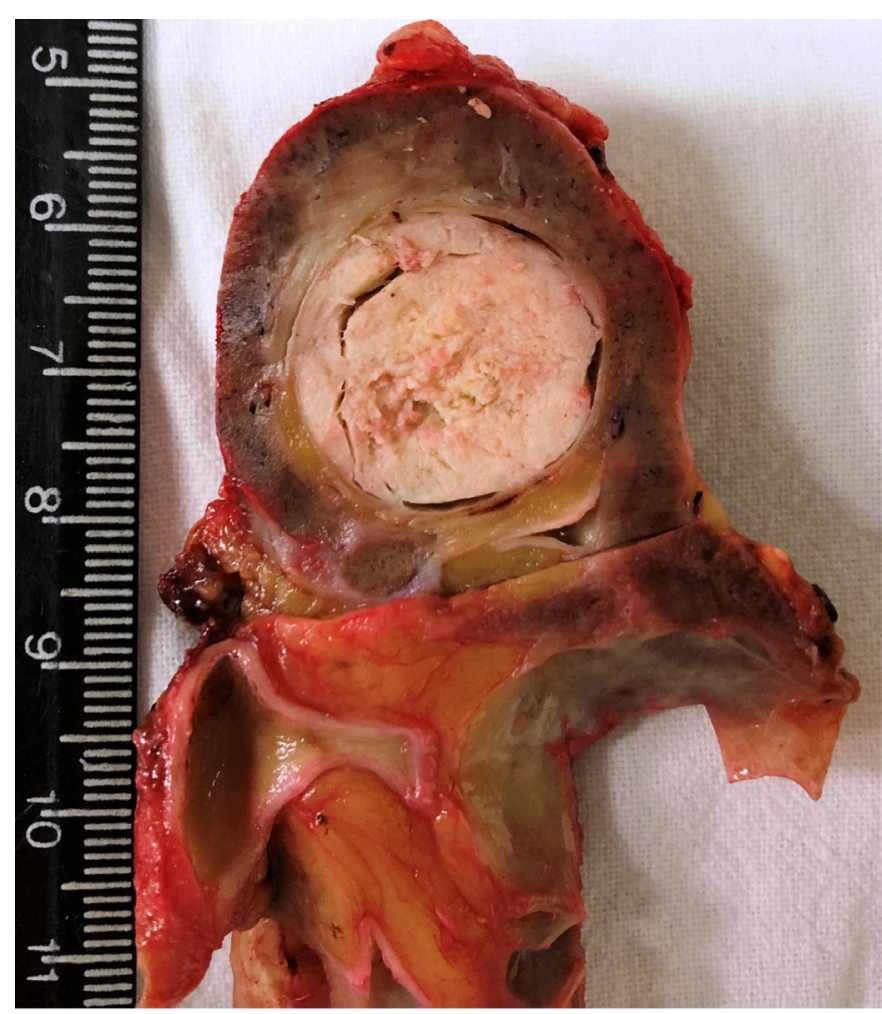

Figure 1 Gross appearance of MTSCC. The tumor is well-circumcribed with homogeneous, well-circumscribed grayish-white cut surface. Notice the atrophic parenchima.

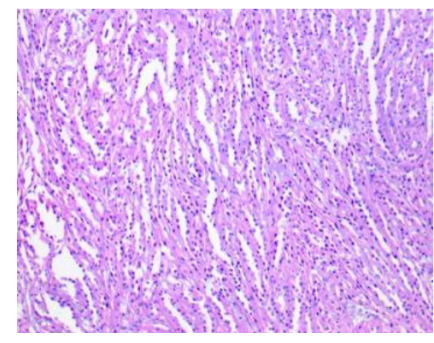

Figure 2A. The neoplasm is constituted by long tubules and spindle cells that lay on a basophilic extracellular mucinous stroma.

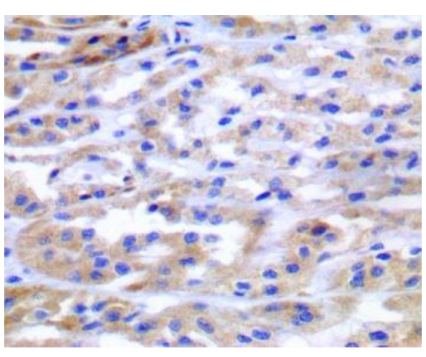

Figure 2E. The tubule and spindle cells tumour were both positive for AMACR pathological findings sustained the diagnosis of a mucinous tubular and spindle cell carcinoma. The patient has been on annual followup for 5 years and imaging exams have not detected signs of relapsed disease or metastasis.

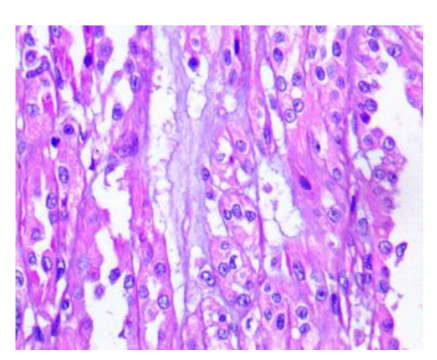

Figure 2B. Low nuclear grade cells with focally vacuolated eosinophilic cytoplasm. Note the mucinous stroma (arrow).

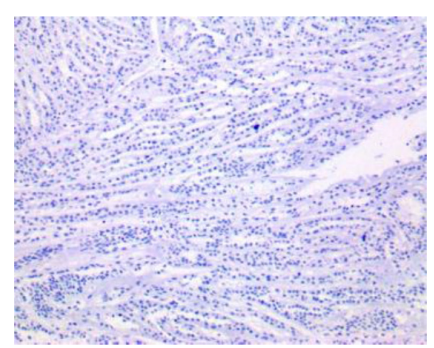

Figure 2F. negative for CD10.

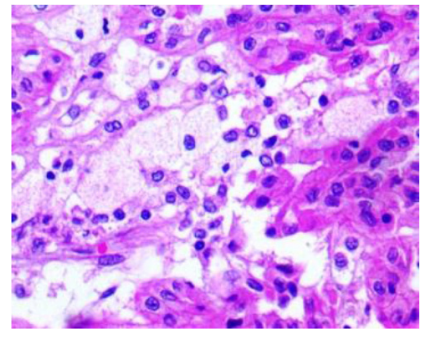

Figure 2C. Some clusters of foamy macrophages were present

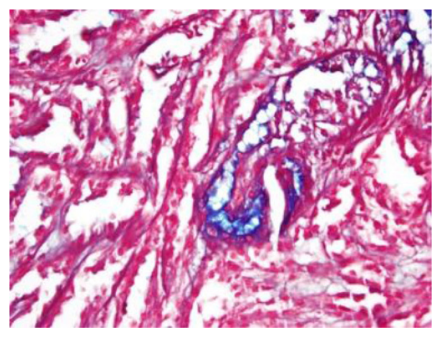

Figure 2D. Extracellular mucin highlighted by Alcian blue stain.

Figure 2. Microscopic appearance of MTSCC. 


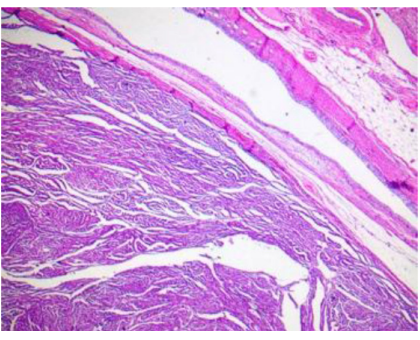

Figure 3A. The tumor is wellcircumscribed.

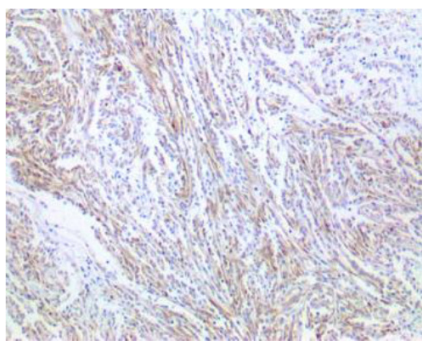

Figure 3E. The tubule and spindle cells tumour were both positive for CK7

Figure 3. Microscopic features of MTSCC.

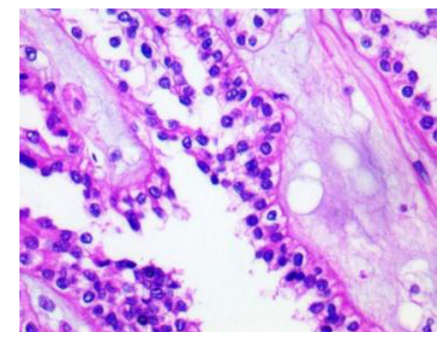

Figure 4A. Stroma is myxoid and bubbly.

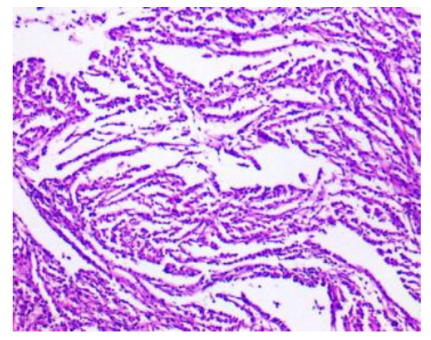

Figure 3B. Note the tubular or cord-like growth pattern.

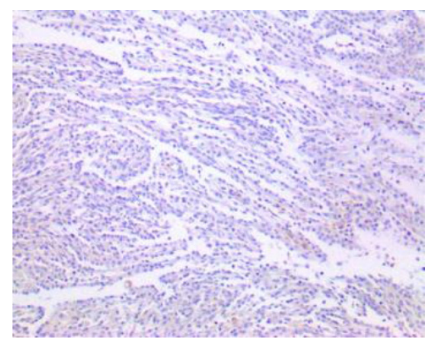

Figure 3F. negative for vimentin.

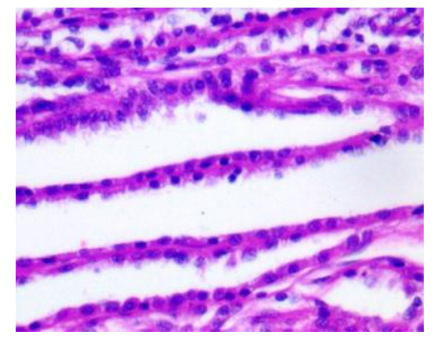

Figure 3C. Fuhrman grade 1 nucleus cuboidal and spindle cells.

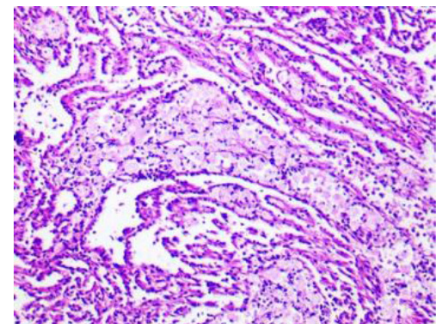

Figure 3D. Foamy macrophages were present in the stroma.

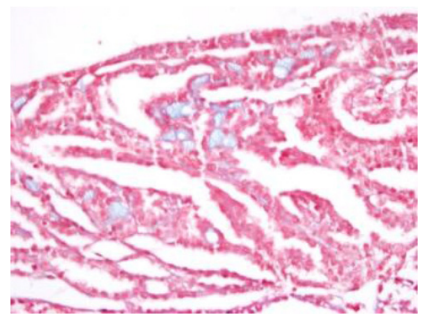

Figure 4B. Alcin Blue stain highlights the stroma.
Figure 4. Stroma microscopic findings.

Table 1. Immunophenotypic Profile of Renal Mucinous Tubular and Spindle Cell Carcinoma

\begin{tabular}{|l|c|c|c|c|c|}
\hline & VIM & CD10 & CK7 & HMWCK & AMACR \\
\hline Case 1 & - & - & + & + & + \\
\hline Case 2 & + & - & + & + & + \\
\hline
\end{tabular}

VIM, vimentin; HMWCK, high-molecular weight cytokeratin; AMACR, alpha-methyl CoA racemase

\section{Discussion}

We present two cases of mucinous tubular and spindle cell renal cell carcinoma affecting two elderly women. MTSCC has a wide age distribution which ranges between 13 to 82 years old and an average of 53 years old. There are a female predominance and a 1: 4 man to women ratio $[4,6,13,14,15]$.

MTSCC is a renal epithelial neoplasm characterized by tubular formations merging with bland spindle cells and a myxoid stroma $[5,16]$. Proper histological classification is essential for a better understanding of this tumor which has already been characterized and distinguished from other renal cell carcinomas (RCC) through immunohistochemical and cytogenetic studies [17]. The vast majority of these renal neoplasms are low grade [13]. However, sarcomatoid differentiation implies in a deleterious prognosis and requires a closer clinical and radiological follow-up [18]. Clinically, most patients present with asymptomatic masses found accidentally by ultrasound [19]. Even though, some MTSCC can manifest as flank pain, hematuria, abdominal mass and systemic repercussions such as fever, anorexia and weight loss $[4,15,1820]$.

On gross examination, mostly occur as a solid mass in the renal cortex and are partially encapsulated. The cut surface is usually shiny, bulging and mucoid and shows pale, grey or homogenous tan colors $[5,6,14]$.

Microscopic sections feature a renal epithelial neoplasm with variable components of tubular or cord-like growth architecture, spindle cell areas and extracellular basophilic mucinous stroma $[6,10,13]$. Necrosis, mucin-poor stroma, cellular pleomorphism, and high nuclear grade are uncommon. Hardly, MTSCC can present aggressive local growth and spread distant metastasis to liver and MTSCC splits into two categories: classic (abundant extracellular mucin stroma) and mucin-poor (little or no extracellular mucin stroma). Mucinous stroma stains blueish to Alcian blue and colloidal iron $[4,14]$.

Immunohistochemical studies confirm that both cuboidal and spindle cells are positive for PAX2/8, epithelial membrane antigen (EMA), low molecular weight cytokeratins (CK8/18, CK19, and CK7), E-cadherin and AMACR. Vimentin and high molecular weight cytokeratin (34BE12) staining show variable expressions. retroperitoneal lymph nodes $[6,14,20]$. Based on extracellular mucin, 
CD10, CD15, and RCC marker are usually positive in other RCC variants and frequently negative in MTSCC $[4-6,8,9,14,15,18]$. Immunohistochemical characteristics of both cases we reported are summarized in table 1 .

Classic MTSCC offers no diagnosis challenges. On the other hand, mucin-poor MTSCC is often misdiagnosed as mesenchymal neoplasms (e.g. inflammatory myofibroblastic tumor) which tend to present elongated nuclei, distinct fascicular arrangement and rarely label cytokeratins strongly. Papillary RCC is another differential diagnosis to be considered which usually has round cells, variable atypia and predominant tubulopapillary pattern and no extracellular mucin. Immunocytochemistry is usually useless [13,15,21]. Classical morphology and complete surgical excision correlate with favorable prognosis. Nevertheless, sarcomatoid features must be reported because they may indicate an aggressive course $[5,6,10,15]$. A close follow-up is recommended for all MTSCC [5,6].

\section{Conclusion}

In summary, we report two cases of mucinous tubular and spindle cell renal cell carcinoma. We consider MTSCC to be a low-grade carcinoma. Although the follow-up for our two cases was favorable, precaution is warned given the small number of reported MTSCC and the fact that some have metastasized. The current study also highlights that additional clinicopathological expertise with these renal tumors is necessary to comprehend their biological behavior.

\section{Authorship}

Queiroz, GA and Silva, WNT collected data, reviewed the literature, analyzed the data and wrote the manuscript; Sudo, RSL and Oliveira, TJ participated in the literature review and helped to write the manuscript; Dornelas, $\mathrm{BC}$ designed the project, collected data, reviewed the literature and revised the manuscript. All authors have read and approved the final manuscript.

Funding information: The authors declare that they have no funding

Units of Measurement: Measurements of length: centimeter $(\mathrm{cm})$

\section{Abbreviations \& Symbols}

MTSCC: mucinous tubular and spindle cell carcinoma

WHO: World Health Organization

RCC: renal cell carcinomas

VIM: vimentin

HMWCK: high-molecular weight cytokeratin

AMACR: alpha-methyl CoA racemase

\section{References}

1. Lopez-Beltran, Scarpelli M, Montironi R, Kirkali Z. (2006) 2004 WHO classification of the renal tumors of the adults. European urology 5: 798-805.

2. Moch H, Cubilla A. L, Humphrey P. A, Reuter V. E, Ulbright T. M. (2016) The 2016 WHO classification of tumours of the urinary system and male genital organs. European urology 1: 37.

3. Trabelsi A, Stita W, Yacoubi M. T, Rammeh S, Hmissa S, et al. (2008) Renal mucinous tubular and spindle cell carcinoma. Canadian Urological Association Journal 6: 635 .
4. Sun N, Fu Y, Wang Y, Tian T, An W, et al. (2013) Mucinous tubular and spindle cell carcinoma of the kidney: A case report and review of the literature. Oncology letters 3: 811-814.

5. Zhao M, He X. L, Teng X. D. (2015). Mucinous tubular and spindle cell renal cell carcinoma: a review of clinicopathologic aspects. Diagnostic pathology 1: 168.

6. Nathany S, Monappa V. (2019) Mucinous Tubular and Spindle Cell Carcinoma: A Review of Histopathology and Clinical and Prognostic Implications. Archives of pathology \& laboratory medicine.

7. Chen Q, Gu Y, Liu B. (2015) Clinicopathological characteristics of kidney mucinous tubular and spindle cell carcinoma. International journal of clinical and experimental pathology 1: 1007.

8. Shen S, Ro J. Y, Tamboli P, Truong L. D, Zhai Q, et al. (2007) Mucinous tubular and spindle cell carcinoma of kidney is probably a variant of papillary renal cell carcinoma with spindle cell features. Annals of diagnostic pathology 1: 13-21.

9. Kuroda N, Nakamura S, Miyazaki E, Hayashi Y, Taguchi, T, et al. (2003) Lowgrade tubular-mucinous renal neoplasm with neuroendocrine differentiation: A histological, immunohistochemical and ultrastructural study. Pathology international 3: 201-207.

10. Rakozy C, Schmahl G. E, Bogner S, Störkel, S. (2002) Low-grade tubularmucinous renal neoplasms: morphologic, immunohistochemical, and genetic features. Modern Pathology 11: 1162.

11. Marks-Jones D. A, Zynger D. L, Parwani A. V, Cai, G. (2009) Fine needle aspiration biopsy of renal mucinous tubular and spindle cell carcinoma: report of two cases. Diagnostic cytopathology 1: 51-55.

12. Pacchioni D, Volante M, Casetta G, Sapino A, Marchiò C, et al. (2007) Myxoid renal tumor with myoepithelial differentiation mimicking a salivary gland pleomorphic adenoma: description of a case. The American journal of surgical pathology 4: 632-636.

13. Owens C L, Argani P, Ali S. Z. (2007) Mucinous tubular and spindle cell carcinoma of the kidney: cytopathologic findings. Diagnostic cytopathology 9: 593-596.

14. Ursani N. A, Robertson A. R, Schieman S. M, Bainbridg T, Srigley J. R. (2011) Mucinous tubular and spindle cell carcinoma of kidney without sarcomatoid change showing metastases to liver and retroperitoneal lymph node. Human pathology 3 : $444-448$.

15. Du J. H, Zhang L, Liang Z. (2018) Huge mucinous tubular and spindle cell carcinoma of kidney: A rare case report and literature review. Medicine 43.

16. Eble, J. N. (2004) World Health Organization classification of tumours. Pathology and Genetics. Tumours of the Urinary System and Male Genital Organs 10.

17. Ferlicot S, Allory Y, Compérat E, Mege-Lechevalier F, Dimet S, et al. (2005) Mucinous tubular and spindle cell carcinoma: a report of 15 cases and a review of the literature. Virchows Archiv 6: 978-983.

18. Simon R. A, di Sant'Agnese, P. A, Palapattu, G. S, Singer, E. A, Candelario, et al. (2007) Mucinous tubular and spindle cell carcinoma of the kidney with sarcomatoid differentiation. International journal of clinical and experimental pathology 2: 180

19. Eble JN and Young RH (2007) Tumors of the urinary tract. In: Diagnostic Histopathology of Tumors. Fletcher CDM (ed), 3rd edition. Churchill Livingstone, Edinburg 537.

20. Parwani A. V, Husain A. N, Epstein J. I, Beckwith J. B, Argani, P. (2001) Lowgrade myxoid renal epithelial neoplasms with distal nephron differentiation. Human pathology 5: 506-512.

21. Fine S. W, Argani P, DeMarzo A. M, Delahunt, B, Sebo, et al. (2006) Expanding the histologic spectrum of mucinous tubular and spindle cell carcinoma of the kidney. The American journal of surgical pathology 12: 1554-1560.

\section{Citation:}

Giulia de Assis Queiroz, Rafael Shiguetaro Lemos Sudo, Thales Junqueira Oliveira, William Nicoleti Turazza da Silva, Bruno de Carvalho Dornelas, Bruno de Carvalho Dornelas (2019) Renal Mucinous Tubular and Spindle Cell Carcinoma: A Report of Two Cases and Literature Review. Cancer Stud Ther $J$ Volume 4(4): 1-4. 